\title{
EDITORIAL
}

\section{ANALYSIS, EVALUATION, AND MANAGEMENT OF OUEUING SYSTEMS}

Ever since Agner Krarup Erlang published the first paper on queuing in 1909, the study of queues has embraced several application areas, including manufacturing/production, inventory management, transportation and logistics, revenue management, and, more recently, service systems such as call centers and health care operations. As application areas continue to grow, the problems studied are becoming increasingly complex. This has led to the development of innovative methodologies or innovative uses of existing methodologies to meet many new challenges. This special issue of Probability in the Engineering and Informational Sciences (PEIS) explores a number of topical issues related to analysis, evaluation, and management of queuing systems. The articles included can be divided into several areas:

- Articles evaluating the key performance of a queuing system or developing methodology to evaluate key performance measures

- Articles on the cost of modeling abstraction; those estimating the negative impact on system performance when the variability of input parameters is ignored

- Articles on the design and control of queuing systems

In the first category, Walraevens, Fiems, and Moeneclaey develop a simple method to approximate the transient behavior of a queuing system via generating functions. Using singularity analysis, the article proposes a method for constructing an asymptotically exact approximation of transient behavior and applies to several different queuing systems.

Along the same lines, Sherman, Kharoufeh, and Abramson consider a queuing model arising in streaming multimedia applications. The authors consider the analysis and control of an $M / G / 1$ retrial queue with an infinite capacity for the retrial queue and normal queue where the decision-maker can control the retrial rate and the server can control the repair rate. The article examines stability conditions and derives key queuing performance measures including the queue length distributions. 
Limón-Robles and Wortman explore an analytical model for studying the timedependent occupancy distribution for a $G / G / 1$ queue and develop tight bounds on the occupancy distribution. As there is no closed form available in the literature, this article offers "near-closed forms" that can be used to determine the occupancy distribution with minimal computational burden.

The article by Winands, Adan, van Houtum, and Down analyzes the queue length and sojourn time distributions of a two-queue model with state-dependent setups. A single server exhaustively serves the high-priority queue and use the $k$-limited strategy for the low-priority queue. The result of the article is applied to evaluate a multiclass stochastic production system. Collectively, these four articles cover the first type of queuing analysis.

In this issue there is but one article that falls in the second category, but we feel it is an important one, especially in light of the keen interest in design and management of call centers. Steckley, Henderson, and Mehrotra investigate the impact of forecast errors in the arrival rate of customers and show that ignoring forecast errors can have a significantly negative impact on predictions of long-run performance. The article shows that, in the presence of the arrival rate variability, more flexible staffing strategies are more appropriate than traditionally accepted fixed-level staffing rules.

The remaining four articles focus on the control queuing systems. Argon, Ding, Glazebrook, and Ziya propose two heuristics based on a dynamic index to route traffic to multiple servers under general delay cost functions. An extensive numerical study shows that both heuristics outperform greedy and min-drift policies in a range of traffic conditions.

Hampshire, Jennings, and Massey consider staffing and provisioning decisions for a call center with a finite buffer and time-varying arrival rates. The article casts the fluid scheduling problem with service-level agreements as a calibrated fluid scheduling problem with appropriate penalties provides insights and intuition into the optimal staffing and provisioning rules. The authors also draw a connection with open-loop control problems in queuing and classical fluid mechanics.

Hampshire, Massey, and Wang examine dynamic pricing policies for time-varying loss systems that maximize revenue while meeting quality of service targets. The dynamic optimization problem takes into account future congestion introduced by an arriving customer. The solution reflects the opportunity cost when setting the price.

Finally, Gayon, Talay-Değirmenci, Karaesmen, and Örmeci consider the effect of pricing strategies in a stochastic production system in a fluctuating demand environment. The article compares three pricing strategies and presents structural properties of optimal production policies.

We believe that, collectively, the articles in this issue represent examples of the state of the art in queuing analysis. We hope that they will serve as a valuable reference for researchers and practitioners. 